\title{
Growing the seeds sown by Piero Sensi
}

\author{
Enrico Selva \\ Piero Sensi is probably known primarily for his role in the discovery of rifamycin and for developing it to be a drug of \\ fundamental importance in the treatment of tuberculosis. He has also contributed to promote screening programs of microbial \\ products and research approaches for antibacterial agents that have been further developed up to the present day. This paper \\ reports a sequence of discovery approaches, failures and successes that spans for about $\mathbf{5 0}$ years and is still in progress. \\ The Journal of Antibiotics (2014) 67, 613-617; doi:10.1038/ja.2014.110; published online 13 August 2014
}

For about three decades Piero Sensi had key scientific and managerial roles at the Lepetit laboratories where rifamycins were discovered and developed. When I joined the microbial products group in the mid70s, I found a scientific and human environment shaped by his guidance. The group was composed of scientists skilled in disciplines as diverse as soil microbiology, screening, fermentation, purification of broth components and medical microbiology, all working at the same site and encouraged to exchange information both in formal and informal ways. Looking back, I feel that this organization has deeply influenced the way in which discovery projects were developed at Lepetit and then in the groups that derived from it. Like many other pharmaceutical companies over the past decades, Lepetit followed a tortuous path of acquisitions and mergers. It first became a part of the Dow Chemical Pharma unit and went through subsequent mergers to become Marion Merrel Dow and then Hoechst Marion Roussel. Then through a management buyout, the natural product unit founded the biotech company, Biosearch Italia that morphed into Vicuron by merger with Versicor. Vicuron was acquired by Pfizer and, although $\mathrm{R}$ and $\mathrm{D}$ was shut down, two new independent research groups were formed by former Vicuron staff: Ricerca per la Vita and Naicons.

Over time the discovery capacity, based on natural products and screening, was applied to diverse therapeutic areas, but a constant focus was maintained on infections caused by bacterial pathogens. Dedicated to Piero Sensi, I try to capture hereafter the principal steps of a multifaceted story of antibacterial screening projects that were developed by the numerous scientists who worked with Piero Sensi and then continued in his footsteps.

By the early 60 s it became evident that the screening of Streptomycetes was decreasing in productivity because of high rate of isolation of known antibiotics and declining clinical potential of the resulting discoveries. An approach to shift to less-exploited microorganisms was an attempt to circumvent these issues . The SheringPlough group focused on Micromonospora ${ }^{1}$ and at Lepetit the efforts were initially directed to the genus Thermoactinomyces. The novel protein synthesis inhibitor thermorubin was soon discovered. ${ }^{2}$
It showed a good activity against bacterial pathogens but was highly antagonized by serum and was inactive in septicemia models. After repeated isolation of thermorubin and of other antibiotics with poor antimicrobial activity, it became clear that Thermoactinomyces had limited potentials. New genera were searched by thorough inspection of isolation plates under the microscope and by using a micromanipulator to pick off those colonies with unusual morphologies. In the years 1966-1967, this labor-intensive approach led to the isolation of new genera, such as Dactylosporangium, Planomonospora, Microtetraspora and Planobispora, ${ }^{3-6}$ that ultimately showed good productivity in antimicrobial screenings. At that time, however, the number of strains obtained was too low to generate a steady flow of discoveries. A new and impressive course ensued when massive isolation of Actinoplanes was achieved by exploiting their spore motility. Soil samples were suspended in water and centrifuged at a low speed. Mobile spores then migrated to the supernatant and were collected. The growth of common bacteria with mobile spores was controlled with the use of antibiotics, in particular with novobiocin that is typically inactive against Actinoplanes. Thousands of Actinoplanes strains were soon available for screening, providing a competitive edge for discovery. Purpuromycin was initially discovered $^{7}$ but showed signs of potential toxicity. Its novel structure, however, confirmed the capacity of Actinoplanes to produce novel secondary metabolites. Having a rich flow of activities coming from Actinoplanes made it possible, and necessary, to focus only on the more promising ones; those that showed attractive features with respect to mechanism of action, spectrum of antibacterial activity, selectivity against prokaryotes vs eukaryotes and efficacy in experimental mouse septicemia.

A method to measure the incorporation of radiolabeled precursors into the main cell macromolecules (RNA, DNA, proteins and peptidoglycan) was set up. These experiments provided an insight as to the metabolic pathway that was primarily inhibited by the antibiotic under investigation. This allowed a focus on antibiotics with specific mechanisms of action, thus anticipating an approach that subsequently became widely used throughout the industry. 
A novel inhibitor of bacterial RNA polymerase was discovered, ${ }^{8}$ named lipiarmycin, because the producer was isolated on February 29 of the leap year 1972. The antibiotic showed good activity against Streptococcus mutans and was considered for development as an antiplaque agent, but was soon discontinued for commercial reasons. Years later lipiarmycin A3, a component of the lipiarmycin mixture produced by Actinoplanes deccanensis, ${ }^{9}$ was rediscovered and given the name tiacumycin B (fidaxomicin), which has been recently approved for Clostridium difficile-associated diarrhea that has become the leading hospital-acquired infection. ${ }^{10}$ Oral fidaxomycin reaches high fecal concentrations, targeting $C$. difficile with low impact on normal fecal bacterial flora and is effective with reduced recurrency rate. ${ }^{11}$

Antibiotic L13365, ${ }^{12}$ also named luxomycin for its characteristic fluorescence, was found to inhibit protein synthesis. It showed good in vitro and in vivo activity but also cross-resistance to nosiheptide and thiostrepton and no advantages over these thiopeptide antibiotics (unpublished data). The novel protein synthesis inhibitor A21459 was also discovered from Actinoplanes. ${ }^{13}$ The antibiotic was not developed because it was ineffective in vivo and showed an unusual and restricted spectrum of antibacterial activity. Some Gram-negative pathogens were sensitive. The option to improve cell penetration in important pathogens by chemical modifications was considered but not implemented because spontaneous mutations to resistance were found to be mediated by the protein synthesis apparatus.

In the course of the screening of Actinoplanes, a screening method was also developed to specifically identify cell wall inhibitors. It was based on its activity against parental Staphylococcus aureus but not against its cell wall lacking isogenic L-form, which is capable of growing in isotonic media. ${ }^{14}$ Three novel antibiotics, gardimycin, techomycin and ramoplanin, were identified by this screen.

Gardimycin (actagardine) was a novel lantibiotic. The lantibiotics are peptides that are ribosomally synthetized and post-translationally modified and that show varied activities. ${ }^{15}$ Gardimycin was the first lantibiotic found to be active primarily on cell wall biosynthesis, ${ }^{14,16}$ analogous to mersacidin that was discovered about two decades later. ${ }^{17}$ Gardimycin showed relatively low in vitro activity but was remarkably more active in mouse septicemia caused by Streptococci compared with that predicted on the basis of its in vitro activity. ${ }^{18}$

Teichomycin (teicoplanin) was discovered from Actinoplanes teichomyceticus. ${ }^{19}$ It is a glycopetide of the vancomycin class structurally characterized by the presence of fatty-acid residues. In animal models it appeared to have a better pharmacokinetic profile than vancomycin the standard of care in therapy, ${ }^{20,21}$ and in clinical studies it proved to be better tolerated and therapeutically effective when administered once a day. ${ }^{22}$ Such improvements are typical objectives of semisynthetic approaches, but in the case of teicoplanin they were achieved by exploiting the versatility of microorganisms. The antibiotic was introduced in the clinical practice in 1987 and is currently used in several countries to treat serious infections caused by methicillin-resistant $S$. aureus (MRSA). In a timeline perspective, teicoplanin represents one of the few cases of novel microbial products that has been promoted for clinical use after the unique post-war period that saw the introduction of the major classes of antibiotics. $^{23}$

The third cell wall inhibitor was ramoplanin (A16686), ${ }^{24}$ which is a cyclic depsipeptide with sugars and acyl chain residues. Ramoplanin showed good bactericidal activity against Gram-positive pathogens, including MRSA and vancomycin-resistant Enterococci (VRE), and efficacy in mouse septicemia. Its parenteral administration was, however, problematic because of severe toxicity at the site of injection. The antibiotic was evaluated for topical use and to control VRE colonizing the gastrointestinal tract. ${ }^{25}$ Because of its high activity against anaerobes it has been more recently developed up to phase-III clinical trials for C. difficile-associated diarrhea. ${ }^{10,26}$

These discoveries occurred over a few years in the $70 \mathrm{~s}$, when Actinoplanes provided numerous and potentially unexploited activities and the screening group focused on the more interesting ones by testing systematically crude broth extracts for mechanism of action and in vitro and in vivo potency. The early determination of in vivo efficacy of gardimycin, teicoplanin and ramoplanin concentrated the efforts on these activities and then revealed underlying characteristics, such as prolonged serum levels in the case of teicoplanin and rapid cidal activity in the case of ramoplanin, that were key properties for their development.

At the beginning of 80 s, the screening focused on sexually transmitted infections because their incidence was increasing at a time when the principal infections appeared under control by the array of antibiotics then available. A spectrum of activity restricted to the specific pathogens was considered to be appropriate for sexually transmitted infections. The screen was thus set up for antibiotics that were active against Neissera gonorrhoeae and inactive against $S$. aureus. This approach selected antibiotics of the class of kirromycin. ${ }^{27}$ These antibiotics, known also as elfamycins, inhibit bacterial protein synthesis acting on elongation factor $\mathrm{Tu}(\mathrm{Ef}-\mathrm{Tu})$ and are inactive against $S$. aureus because its Ef-Tu is naturally insensitive. ${ }^{28} \mathrm{~A}$ new elfamycin was discovered ${ }^{29}$ but the project was abandoned as the antibiotics selected in the course of the screening showed low serum levels in animal models when given intramuscularly or orally.

In the meantime, novel screening approaches were developed based on molecular interactions between targets and antibiotics. The group was then developing the glycopeptide teicoplanin and was clearly interested in its binding to the $\mathrm{D}$-ala-D-ala terminus of peptidoglycan precursor. A synthetic D-ala-D-ala residue was coupled to a polymer to make an affinity resin. ${ }^{30}$ Glycopeptides were captured by affinity on the resin and eluted at basic pH. Fermentation broths were thus applied to micro columns of the resin and the antimicrobial activities that were retained and eluted as the standard glycopeptides were selected. Quickly, > 70 glycopetides were detected ${ }^{31}$ many with novel structures, such as antibiotic A42867. ${ }^{32}$ It resulted that the glycopetide A40926 produced by Actinomadura sp. had good antibacterial activity, including $N$. gonorrhoeae, and showed serum levels higher and more prolonged than teicoplanin. ${ }^{33}$ Although it was decided to keep the company focused on the leading teicoplanin, A40926 was patented. This pre-dated the discovery of parvodicin, ${ }^{34}$ an identical antibiotic independently isolated in the Smith Kline and French laboratories, also using a screening method based on affinity. ${ }^{35}$ Dalbavancin is a semisynthetic derivative of A40926 with very long-lasting serum levels that allow a once-a-week treatment. It has been recently approved by the US Food and Drug Administration for the treatment of acute skin MRSA infections.

On the basis of the experience with kirromycins and glycopeptides, a method was developed to capture by affinity novel inhibitors of Ef-Tu. An agar diffusion test on S. aureus was devised to search activities reversed by the presence of exogenous Ef-Tu. ${ }^{36}$ The novel thiazolyl peptide antibiotic GE2270 was discovered from Planobispora sp., ${ }^{37}$ followed by the structurally related GE37468. ${ }^{38}$ The activity of GE2270 on Ef-Tu was confirmed by their mechanism of action studies. ${ }^{39}$ The antibiotic showed potent antimicrobial activity against Gram-positive pathogens, including MRSA and VRE, and good efficacy in experimental infections. It turned out, however, to be difficult to formulate in a clinically acceptable form. Chemical modifications were attempted with partial success. The project has 
been further developed by scientists in the Novartis laboratories to generate the LFF-571 derivative that is currently in phase-II clinical trials. ${ }^{10}$

In the 90s, novel cell-free assays were introduced on the assumption that they were more sensitive than the classical screening tests and thus capable of detecting activities produced at low concentration as well as novel inhibitors of intracellular targets but impaired in cell penetration and thus not detectable as antibacterials. The assumption was that chemical modifications could improve cell penetration and make them antibacterial agents.

Screening with a cell-free test on a rifampicinR RNA polymerase led to the discovery of the novel inhibitor GE23077 produced by an Actinomadura sp. ${ }^{40,41}$ GE23077 is a cyclic heptapeptide with potent activity against RNA synthesis in cell-free tests, but with antibacterial activity restricted to Moraxella catarrhalis. The molecule was modified chemically to improve cell penetration but with limited results.

A cell-free bacterial protein synthesis system ${ }^{42,43}$ was tried out subsequently. As consequence of the failure to make GE23077 permeable, it was decided to follow the inhibitors of the cell-free assay only if they show some antibacterial activity on whole cells, thus exhibiting some ability to penetrate bacteria cells. The novel antibiotics GE81112, ${ }^{42,44}$ orthoformimycin ${ }^{45}$ and GE82832 ${ }^{46-48}$ were thus discovered and found to inhibit bacterial protein synthesis by different and novel mechanisms. ${ }^{42-48}$

In the course of the years the entire set of operations of the screening process was adapted to the emerging needs. It was observed that the antibiotics discovered in the 60 s and 70 s were frequently produced in remarkably high yield by the wild-type strains. More sensitive assays were introduced to detect minor products. To get enough bioactive material for initial characterization, it was necessary to scale up fermentation, as soon as possible, to 2001 and to use styrenic adsorption resins in the first step of purification to reduce the volumes from the fermentation plant to the lab scale. The method based on styrenic resin proved convenient because it recovered more water-soluble activities with respect to the then conventional extraction with water-immiscible solvent, and was effective in eliminating components such as salts and lytic enzymes that typically interfere in cell-free assays. The process was thus routinely used to prepare on a micro scale extracts appropriate for an increased variety of screening assays. In addition, investigations of the activities emerging from screening was improved by using HPLC fractionations with MS detection and biological testing of the eluted fractions ${ }^{49}$ and by systematic comparison with a proprietary database of the microbial products reported in the literature and patents. ${ }^{50}$

Starting from late 90s, a collection of broth extracts was generated for high-throughput screening. At maturity the collection was composed of $>160000$ extracts derived from a collection of $>55000$ actinomycetes and fungi. ${ }^{51}$ Each extract was a complex mixture of molecules. In the course of routine investigations on the extracts it was observed that the bioactivity was frequently associated with peaks of minor intensity in the HPLC chromatograms This complexity, although demanding dedicated work for the processing of the activities selected, was providing broad chemical diversity. By examining the collection from the antiifective standpoint, thousands of extracts were found capable of inhibiting Gram-positive and -negative test bacteria. ${ }^{51}$ This abundance compares favorably with the limited occurrence of antimicrobial compounds observed by screening synthetic products libraries. ${ }^{52}$

This new collection of extracts was tested for inhibitors of cell wall synthesis on the basis of its activities on S. aureus and its L-form. After a counter-screen to remove classical beta-lactams and gycopeptides, several gardimycin-type lantibiotics were identified. ${ }^{53,54}$ The more interesting in term of potency and antibacterial spectrum was the antibiotic microbisporicin (1 07 891; NAI-107) from Microbispora sp. ${ }^{55}$ In preclinical studies it showed good activity against Gram-positive bacteria including MRSA, glycopeptide-intermediate

Table 1 Main discoveries

\begin{tabular}{|c|c|c|c|c|c|}
\hline Antibiotic & Producer (genus) & Target & Structural characteristics & Year & Reference \\
\hline Thermorubin & Thermoactinomyces & Protein synthesis & Aromatic polyketide & $1962^{\mathrm{a}}$ & 2 \\
\hline Purpuromycin & Actinoplanes & Protein synthesis-tRNA & Spiroketal compound & $1973^{a}$ & 7,74 \\
\hline Lipiarmycin & Actinoplanes & RNA polymerase & Macrolactone & $1973^{a}$ & 8 \\
\hline Actagardine (Gardimycin) & Actinoplanes & Cell wall synthesis & Lantibiotic & $1974^{a}$ & 14,16 \\
\hline Teicoplanin (Teichomycin) & Actinoplanes & Cell wall synthesis & Glycopeptide & $1975^{a}$ & $19-22$ \\
\hline L13365 (Luxomycin) & Actinoplanes & Protein synthesis & Thiazolyl peptide & $1976^{\mathrm{a}}$ & 12 \\
\hline Ramoplanin (A16686) & Actinoplanes & Cell wall synthesis & Lipoglycodepsipeptide & $1979^{a}$ & 24,58 \\
\hline A21459 & Actinoplanes & Protein synthesis & Cyclic peptide & $1980 s^{b}$ & 13 \\
\hline SB22484 & Streptomyces & Protein synthesis -EF-Tu & Elfamycin type & $1983^{a}$ & 29 \\
\hline A40926 & Actinomadura & Cell wall synthesis & Glycopeptide & $1984^{a}$ & 33 \\
\hline A42867 & Nocardia & Cell wall synthesis & Glycopeptide & $1986^{a}$ & 32 \\
\hline GE2270 & Planobispora & Protein synthesis-EF-Tu & Thiazolyl peptide & $1988^{a}$ & 37,39 \\
\hline GE37468 & Streptomyces & Protein synthesis-EF-Tu & Thiazolyl peptide & $1995^{a}$ & 38 \\
\hline GE23077 & Actinomadura & RNA polymerase & Cyclic Peptide & $2000^{a}$ & 40,41 \\
\hline GE81112 & Streptomyces & Protein synthesis & Tetrapetide & $2001^{a}$ & 42,44 \\
\hline Planosporicin (97518) & Planomonospora & Cell wall synthesis & Lantibiotic & $2003^{a}$ & 53,62 \\
\hline Microbisporicin (107891; NAI-107) & Microbispora & Cell wall synthesis & Lantibiotic & $2005^{a}$ & 55,57 \\
\hline GE82832 & Streptosporangium & Protein synthesis & Depsipetide & $2006^{b}$ & $46-48$ \\
\hline NAI-802 (104802) & Actinoplanes & Cell wall synthesis & Lantibiotic & $2011^{\mathrm{a}}$ & 63 \\
\hline NAI-112 (112781) & Actinoplanes & (Cell wall) Neuropathic pain & Lantibiotic & $2012^{a}$ & 51,64 \\
\hline Orthoformimycin (107558) & Streptomyces & Protein synthesis & Orthoformate Compound & $2012^{c}$ & 45 \\
\hline
\end{tabular}

aThe date refers to patent priority date.

bThe date refers to period of investigation.

cThe date refers to date of publication. 
S. aureus and VRE, and efficacy in several models of experimental infection. ${ }^{56}$ The antibiotic inhibits peptidoglycan biosynthesis by acting on lipid-II intermediate ${ }^{57}$ and contains a 5-chlorotryptofan moiety that is unique in the lantibiotic class.

Interestingly, the glycopeptides teicoplanin and A40926, the lipoglyco-depsipeptode ramoplanin and the lantibiotic microbisporicin (107891; NAI-107), that were discovered as cell wall inhibitors, all inhibit in distinct modes the final phase of peptidoglycan synthesis ${ }^{57,58}$ and all contain chlorine in their structures. Their deschloro homologs, when available, were less active against most pathogens with a few exceptions. ${ }^{59-61}$

Other lantibiotics discovered included: Planosporicin (97 518) from Planomonospora sp., ${ }^{53,62}$ NAI-802 (104 802) $)^{63}$ and NAI-112 (1 12 781) both from Actinoplanes. NAI-112 is a particular case. It is a labionincontaining lanthipeptide that shows moderate antimicrobial potency but marked activity on neuropathic pain in animal model. ${ }^{51,64}$

During the years, the screening projects were supported by constantly searching for rare actinomycetes. A novel stimulus came from molecular biology and DNA probing applied to strain characterization and analysis of microbial population in soil habitats. ${ }^{65}$ These efforts allowed to isolate new lineages of Actinomycetales as Catenulispora, Actinospica and Actinoallomurus, ${ }^{66-69}$ which have proved to be capable of producing novel bioactive microbial metabolites $^{70-72}$ and an enzyme of potential medical utility. ${ }^{73}$

Table 1 summarizes the main discoveries that originated from the research efforts that were started by Piero Sensi and have been continued up to the present day. This sequence of discoveries has been promoted by the extraordinary versatility of microorganisms and by the dedication of many scientists. They have survived tortuous and at times critical company events and have experienced both failures and unique successes in the never-ending struggle to combat emerging bacterial pathogens.

1 Weinstein, M. J. Micromonospora antibiotic discovery at Schering/Schering Plough (1961-1973): a personal reminiscence. SIM News 54, 56-66 (2004).

2 Pirali, G., Somma, S., Lancini, G. C. \& Sala, F. Inhibition of peptide chain initiation in Escherichia coli by thermorubin. Biochim. Biophys. Acta 366, 310-318 (1974).

3 Thiemann, J. E., Pagani, H. \& Beretta, G. A new genus of the Actinoplanaceae Dactylosporangium, gen. nov. Arch. Mikrobiol. 58, 42-52 (1967).

4 Thiemann, J. E. \& Beretta, G. A new genus of the Actinoplanaceae: Planobispora, gen. nov. Arch. Mikrobiol. 62, 157-166 (1968).

5 Thiemann, J. E., Pagani, H. \& Beretta, G. A new genus of the Actinomycetales: Microtetraspora gen. nov. J. Gen. Microbiol. 50, 295-303 (1968).

6 Thiemann, J. E., Pagani, H. \& Beretta, G. A new genus of the Actinoplanaceae: Planomonospora gen. nov. G. Microbiol. 15, 27-38 (1967).

7 Coronelli, C., Pagani, H., Bardone, M. R. \& Lancini, G. C. Purpuromycin, a new antibiotic isolated from Actinoplanes ianthinogenes N. sp. J. Antibiot. 27, 161-168 (1974).

8 Coronelli, C., White, R. J., Lancini, G. C. \& Parenti, F. Lipiarmycin, a new antibiotic from Actinoplanes. II. Isolation, chemical, biological and biochemical characterization. J. Antibiot. 28, 253-259 (1975).

9 Cavalleri, B., Arnone, A., Di Modugno, E., Nasini, G. \& Goldstein, B. P. Structure and biological activity of lipiarmycin B. J. Antibiot. 41, 308-315 (1988).

10 Butler, M. S., Blaskovich, M. A. \& Cooper, M. A. Antibiotics in the clinical pipeline in 2013. J. Antibiot. 66, 571-591 (2013)

11 Scott, L. J. Fidaxomicin: a review of its use in patients with Clostridium difficile infection. Drugs 73, 1733-1747 (2013).

12 Pagani, H., Parenti, F., Coronelli, C. \& Tamoni, G. Antibiotic L 13365 produced by Actinoplanes. US4280001A (1981).

13 Selva, E. et al. Antibiotics A21459 A and B, new inhibitors of bacterial protein synthesis. I. Taxonomy, isolation and characterization. J. Antibiot. 49, 145-149 (1996).

14 Somma, S., Merati, W. \& Parenti, F. Gardimycin, a new antibiotic inhibiting peptidoglycan synthesis. Antimicrob. Agents Chemother. 11, 396-401 (1977).

15 Willey, J. M. \& VanDerDonk, W. A. Lantibiotics: peptides of diverse structure and function. Annu. Rev. Microbiol. 61, 477-501 (2007).
16 Coronelli, C., Tamoni, G. \& Lancini, G. C. Gardimycin, a new antibiotic from Actinoplanes. II. Isolation and preliminary characterization. J. Antibiot. 29, 507-510 (1976).

17 Chatterjee, S. et al. Antibiotic, mersacidin, a process for the preparation thereof and the use thereof as a pharmaceutical. US5112806A (1992).

18 Arioli, V., Berti, M. \& Silvestri, L. G. Gardimycin, a new antibiotic from Actinoplanes. III. Biological properties. J. Antibiot. 29, 511-515 (1976).

19 Bardone, M. R., Paternoster, M. \& Coronelli, C. Teichomycins, new antibiotics from Actinoplanes teichomyceticus nov. sp. II. Extraction and chemical characterization. J. Antibiot. 31, 170-177 (1978).

20 Arioli, V., Berti, M. \& Candiani, G. Activity of teicoplanin in localized experimenta infections in rats. J. Hosp. Infect. 7(Suppl A), 91-99 (1986).

21 Pallanza, R. et al. Teichomycin: in-vitro and in-vivo evaluation in comparison with other antibiotics. J. Antimicrob. Chemother. 11, 419-425 (1983).

22 Parenti, F., Schito, G. C. \& Courvalin, P. Teicoplanin chemistry and microbiology. J. Chemother. 12(Suppl 5), 5-14 (2000).

23 Fernandes, P. Antibacterial discovery and development-the failure of success? Nat Biotechnol. 24, 1497-1503 (2006).

24 Cavalleri, B., Pagani, H., Volpe, G., Selva, E. \& Parenti, F. A16686. A new antibiotic from Actinoplanes. Fermentation, isolation and preliminary physicochemical characteristics. J. Antibiot. 37, 309-317 (1984)

25 Montecalvo, M. A. Ramoplanin: a novel antimicrobial agent with the potentia to prevent vancomycin-resistant enterococcal infection in high-risk patients J. Antimicrob. Chemother. 51(Suppl 3), 31-35 (2003)

26 Butler, M. S. \& Cooper, M. A. Antibiotics in the clinical pipeline in 2011. J. Antibiot. 64, 413-425 (2011).

27 Wolf, H. \& Zähner, H. Metabolic products of microorganisms. 99. Kirromycin. Arch Mikrobiol. 83, 147-154 (1972).

28 Hall, C. C., Watkins, J. D. \& Georgopapadakou, N. H. Comparison of the Tu elongation factors from Staphylococcus aureus and Escherichia coli: possible basis for elfamycin insensitivity. Antimicrob. Agents Chemother. 35, 2366-2370 (1991).

29 Selva, E. et al. Antibiotic SB22484: a novel complex of the aurodox group. I. Taxonomy of the producing organism, isolation of the antibiotics and chemical and biological characterization. J. Antibiot. 43, 1349-1358 (1990).

30 Corti, A. \& Cassani, G. Synthesis and characterization of D-alanyl-D-alanine-agarose. A new bioselective adsorbent for affinity chromatography of glycopeptide antibiotics. Appl. Biochem. Biotechnol. 11, 101-109 (1985).

31 Cassani, G. Glycopeptides: antibiotic discovery and mechanism of action. Prog. Ind. Microbiol. 27, 221-235 (1989).

32 Riva, E. et al. A42867, a novel glycopeptide antibiotic. J. Antibiot. 42, 497-505 (1989).

33 Goldstein, B. P. et al. A40926. A new glycopeptide antibiotic with anti-Neisseria activity. Antimic. Agents Chemother. 31, 1961-1966 (1987).

34 Christensen, S. B. et al. Parvodicin, a novel glycopeptide from a new species, Actinomadura parvosata: discovery, taxonomy, activity and structure elucidation. J. Antibiot. 40, 970-990 (1987).

35 Rake, J. B. et al. Glycopeptide antibiotics: a mechanism-based screen employing a bacterial cell wall receptor mimetic. J. Antibiot. 39, 58-67 (1986).

36 Selva, E. et al. Targeted screening for elongation factor Tu binding antibiotics. J. Antibiot. 50, 22-26 (1997)

37 Selva, E. et al. Antibiotic GE2270 A: A novel inhibitor of bacterial protein synthesis. I. Isolation and characterization. J. Antibiot. 44, 693-701 (1991).

38 Stella, S. et al. Antibiotic GE37468 A: a new inhibitor of bacterial protein synthesis I. Isolation and characterization. J. Antibiot. 48, 780-786 (1995).

39 Anborgh, P. H. \& Parmeggiani, A. New antibiotic that acts specifically on the GTP-bound form of elongation factor Tu. EMBO J. 10, 779-784 (1991).

40 Ciciliato, I. et al. Antibiotics GE23077, novel inhibitors of bacterial RNA polymerase. I. Taxonomy, isolation and characterization. J. Antibiot. 57, 210-217 (2004).

41 Sarubbi, E., Monti, F., Corti, E., Miele, A. \& Selva, E. Mode of action of the microbial metabolite GE23077, a novel potent and selective inhibitor of bacterial RNA polymerase. Eur. J. Biochem. 271, 3146-3154 (2004).

42 Brandi, L. et al. Novel tetrapeptide inhibitors of bacterial protein synthesis produced by a Streptomyces sp. Biochemistry 45, 3692-3702 (2006)

43 Donadio, S. et al. Targets and assays for discovering novel antibacterial agents. J. Biotechnol. 99, 175-185 (2002).

44 Brandi, L. et al. Specific, efficient, and selective inhibition of prokaryotic translation initiation by a novel peptide antibiotic. Proc. Natl Acad. Sci. USA 103 39-44 (2006).

45 Maffioli, S. et al. Orthoformimycin, a selective inhibitor of bacterial translation elongation from Streptomyces containing an unusual orthoformate. ACS Chem. Biol. 8, 1939-1946 (2013).

46 Brandi, L. et al. Structural and functional characterization of the bacterial translocation inhibitor GE82832. FEBS Lett. 586, 3373-3378 (2012)

47 Brandi, L. et al. Characterization of GE82832, a peptide inhibitor of translocation interacting with bacterial 30S ribosomal subunits. RNA 12, 62-70 (2006).

48 Bulkley, D. et al. The antibiotics dityromycin and GE82832 bind protein S12 and block EF-G-catalyzed translocation. Cell Rep. 6, 357-365 (2014).

49 Ackermann, B. L., Regg, B. T., Colombo, L., Stella, S. \& Coutant, J. E. Rapid analysis of antibiotic-containing mixtures from fermentation broths by using liquid chromatography-electrospray ionization-mass spectrometry and matrix-assisted lase desorption ionization-time-of-flight-mass spectrometry. J. Am. Soc. Mass Spectrom. 7 , 1227-1237 (1996) 
50 Lazzarini, A., Cavaletti, L., Toppo, G. \& Marinelli, F. Rare genera of actinomycetes as potential producers of new antibiotics. Antonie Van Leeuwenhoek 79, 399-405 (2001).

51 Monciardini, P., Iorio, M., Maffioli, S., Sosio, M. \& Donadio, S. Discovering new bioactive molecules from microbial sources. Microb. Biotechnol. 7, 209-220 (2014).

52 Payne, D., Gwynn, M. N., Holmes, D. J. \& Pompliano, D. L. Drugs for bad bugs: confronting the challenges of antibacterial discovery. Nat. Rev. Drug Discov. 6, 29-40 (2007).

53 Castiglione, F. et al. A novel lantibiotic acting on bacterial cell wall synthesis produced by the uncommon actinomycete Planomonospora sp. Biochemistry 46, 5884-5895 (2007).

54 Jabes, D. \& Donadio, S. Strategies for the isolation and characterization of antibacterial lantibiotics. Methods Mol. Biol. 618, 31-45 (2010).

55 Castiglione, F. et al. Determining the structure and mode of action of microbisporicin, a potent lantibiotic active against multiresistant pathogens. Chem. Biol. 15, 22-31 (2008).

56 Jabés, D. et al. Efficacy of the new lantibiotic NAl-107 in experimental infections induced by multidrug-resistant Gram-positive pathogens. Antimicrob. Agents Chemother. 55, 1671-1676 (2011)

57 Münch, D. et al. The lantibiotic NAI-107 binds to bactoprenol bound cell wall precursors and impairs membrane functions. J. Biol. Chem. 289, 12063-12076 (2014).

58 Reynolds, P. E. \& Somner, E. A. Comparison of the target sites and mechanisms of action of glycopeptide and lipoglycodepsipeptide antibiotics. Drugs Exp. Clin. Res. 16, 385-389 (1990).

59 Maffioli, S. et al. Characterization of the congeners in the lantibiotic NAl-107 complex. J. Nat. Prod. 77, 79-84 (2014).

60 Malabarba, A. et al. Dechloro teicoplanin antibiotics. J. Antibiot. 42, 1684-1697 (1989).

61 Beltrametti, F. et al. Production and characterization of monochlorinated and dechlorinated A40926 derivatives. J. Antibiot. 56, 773-782 (2003).
62 Maffioli, S. et al. Structure revision of the lantibiotic 97518. J. Nat. Prod. 72, 605-607 (2009).

63 Simone, M., Monciardini, P., Gaspari, E., Donadio., S. \& Maffioli, S. Isolation and characterization of NAI-802, a new lantibiotic produced by two different Actinoplanes strains. J. Antibiot. 66, 73-78 (2013).

64 lorio, M. et al. A glycosylated, labionin-containing lanthipeptide with marked antinociceptive activity. ACS Chem. Biol. 9, 398-404 (2014).

65 Donadio, S. et al. Microbial technologies for the discovery of novel bioactive metabolites. J. Biotechnol. 99, 187-198 (2002).

66 Cavaletti, L. et al. Actinospica robiniae gen. nov., sp. nov. and Actinospica acidiphila sp. nov.: proposal for Actinospicaceae fam. nov. and Catenulisporinae subord. nov. in the order Actinomycetales. Int. J. Syst. Evol. Microbiol. 56, 1741-1746 (2006).

67 Busti, E. et al. Catenulispora acidiphila gen. nov., sp. nov., a novel, mycelium-forming actinomycete, and proposal of Catenulisporaceae fam. nov. J Nat. Prod. 75, 1044-1050 (2012).

68 Monciardini, P. et al. Novel members of the family Micromonosporaceae, Rugosimonospora acidiphila gen. nov., sp. nov. and Rugosimonospora africana sp. nov. Int. J. Syst. Evol. Microbiol. 59, 2752-2758 (2009).

69 Pozzi, R. et al. The genus Actinoallomurus and some of its metabolites. J. Antibiot. 64, 133-139 (2011).

70 Busti, E. et al. Antibiotic-producing ability by representatives of a newly discovered lineage of actinomycetes. Microbiology. 152, 675-683 (2006).

71 lorio, M. et al. Chrolactomycins from the actinomycete actinospica. J. Nat. Prod. 75 , 1991-1993 (2012).

72 Mazzetti, C. et al. Halogenated spirotetronates from Actinoallomurus. J. Nat. Prod. 75, 1044-1050 (2012).

73 Cavaletti, L., Carrano, L., Abbondi, M., Brunati, M. \& Taravaglia, A. New protease able to hydrolyze gluten proteins at acidic $\mathrm{pH}$, from the Actinomycete Actinoallomorus. International Patent Application PCT/EP2012/071816 (2013).

74 Kirillov, S. et al. Purpuromycin: an antibiotic inhibiting tRNA aminoacylation. RNA 8, 905-913 (1997). 